Pacific Journal of Mathematics

EVALUATION SUBGROUPS OF FACTOR SPACES 


\title{
EVALUATION SUBGROUPS OF FACTOR SPACES
}

\author{
George E. LANG, JR.
}

In a series of papers Daniel H. Gottlieb defined and studied evaluation subgroups of homotopy groups. In this paper we develop techniques for calculating these subgroups for some factor spaces. The calculations give information on the vanishing of Whitehead products and the existence of cross sections to certain types of fibrations.

The author is indebted to D. H. Gottlieb and J. Siegel for many suggestions during this work.

With the exception of finite topological groups, all spaces are assumed to be locally compact, path connected $C W$ complexes with base point. The base point of spaces $A, B, \cdots, X, Y$ will always be denoted by $a_{0}, b_{0}, \cdots, x_{0}, y_{0}$. When the domain is clear the symbol $x_{0}$ will also denote the constant function with image $x_{0} \cdot 1_{A}$ will denote the identity map from $A$ to $A$ for any set $A$. Homology and cohomology groups are assumed to be singular with integer coefficients. $A \vee B$ and $A \times B$ will denote the one point union and Cartesian products respectively.

The following can be found in [7] or [8] unless otherwise stated.

DEFINITION I.1. The evaluation subgroup $G_{n}(X)$ is the subgroup of $\pi_{n}(X)$ containing all elements $\alpha$ which can be represented by a map $f: S^{n} \rightarrow X$ such that $1_{X} \vee f: X \times S^{n} \rightarrow X$ extends to a map $\phi: X \times S^{n} \rightarrow X$.

The map $\phi: X \times S^{n} \rightarrow X$ will be called an associated map for $\alpha \epsilon$ $G_{n}(X)$.

Let $M$ be the path component of the space of maps from $X$ to $X$ containing the identity map. If $\omega: M \rightarrow X$ is the evaluation map defined by $\omega(f)=f\left(x_{0}\right)$, then $G_{n}(X)=\omega_{*}\left(\pi_{n}(M)\right) \subset \pi_{n}(X) . \quad G_{n}(X)$ is then clearly a subgroup. This alternate definition motivated the name evaluation subgroup.

THEOREM I.2. $G_{n}(X)$ is the set of all $\alpha \in \pi_{n}(X)$ such that there is a fibration $p: E \rightarrow S^{n+1}$ with $X$ as a fiber and $\alpha=\partial\left(\iota_{n+1}\right)$ where $\iota_{n+1}=\left[1_{S^{n+1}}\right] \in \pi_{n+1}\left(S^{n+1}\right)$ and $\partial$ is the boundary homomorphism in the homotopy exact sequence for $p$.

Corollary I.3. If $G_{n}(X)=0$, any fibration with base $S^{n+1}$ and fiber $X$ admits a cross section.

Definition I.4. $P_{n}(X) \subset \pi_{n}(X)$ is the set of elements $\alpha \in \pi_{n}(X)$ 
such that $[\alpha, \beta]=0$ for all $\beta \in \pi_{m}(X)$, all $m$, where [,] denotes the standard Whitehead product.

Theorem I.5. $G_{n}(X) \subset P_{n}(X)$ for all $n$.

Definition I.6. $X$ is said to be a $G$-space if $\pi_{n}(X)=G_{n}(X)$ for all $n$.

Definition I.7. $X$ is said to be a $W$-space if $P_{n}(X)=\pi_{n}(X)$ for all $n$.

It is known [7], that an $H$-space is a $G$-space and clear from Theorem I.5 that a $G$-space is a $W$-space. J. Siegel [13] produced a finite dimensional $G$-space which is not an $H$-space. T. Ganea [5] gave an example of a $W$-space which is not a $G$-space. A finite dimensional $W$-space which is not a $G$-space is given in Section III. B of this paper.

Theorem I.8. $G_{1}(X)$ is contained in the center of $\pi_{1}(X)$.

THEOREM I.9.

$$
P_{n}\left(S^{n}\right)=G_{n}\left(S^{n}\right)= \begin{cases}0 & \text { for } n \text { even } \\ Z & n=1,3, \text { or } 7 \\ 2 Z & n \text { odd }, n \neq 1,3, \text { or } 7 .\end{cases}
$$

II. Factor spaces of topological and Lie groups. In this section machinery is developed for the calculation of certain evaluation subgroups. Unless otherwise stated $Y$ will denote a simply connected topological group and $G$ a finite subgroup. $G$ can be considered as a group of homeomorphisms acting on $Y$ by left multiplication. For $g \in G$ and $y \in Y$ the action will be denoted by $\ell_{g}(y)=g \cdot y$; the orbit an space of this action will be denoted $Y / G$. By 2.7.8 of [15] there is isomorphism $\psi: G \rightarrow \pi_{1}(Y / G)$ and $G$ is the group of covering transformations of the natural covering projection $p: Y \rightarrow Y / G$. For any groups $K$ and $L, Z(K)$ will denote the center of $K$ and $Z_{L}(K)$ will denote the centralizer of $K$ in $L$. Let $e \in G$ denote the identity element and the base point of $Y$. We recall the following theorems from [6].

THEOREM II.1. $G_{1}(X)$ is isomorphic to the subgroup of the covering transformations for the universal covering space which are homotopic to the identity by a fiber preserving homotopy.

Theorem II.2. If $h_{t}: Y \rightarrow Y$ is a homotopy of $1_{Y}, h_{t}$ is fibre preserving if and only if $h_{t}$ commutes with each covering transformation. 
TheOREM II.3. If $g \in G \cap Z(Y)$ then $\psi(g) \in G_{1}(Y / G)$.

Proof. By Theorem II.1 it suffices to show that the covering transformation $\ell_{g}$ is homotopic to $1_{Y}$ by a fiber preserving homotopy. Let $\sigma: I \rightarrow Y$ be a path such that $\sigma(0)=g$ and $\sigma(1)=e$. Consider the homotopy $h_{t}: Y \rightarrow Y$ defined by $h_{t}(y)=y \cdot \sigma(t)$. Then if $\ell_{g}$, is any covering transformation, $h_{t}\left(\ell_{g^{\prime}}(y)\right)=h_{t}\left(g^{\prime} \cdot y\right)=g^{\prime} \cdot y \cdot \sigma(t)=\ell_{g^{\prime}}\left(h_{t}(y)\right)$ and $h_{t}$ is fiber preserving by Theorem II.2. Since $g \in Z(Y)$ by hypothesis, $h_{0}(y)=y \cdot \sigma(0)=y \cdot g=g \cdot y=\ell_{g}(y)$ for all $y \in Y$. But $h_{1}(y)=$ $y \cdot \sigma(1)=y \cdot e=y$, thus $h_{t}$ is the required homotopy and $\psi(g) \in G_{1}(Y / G)$.

THEOREM II.4. If $Z(G)$ lies in a path component of $Z_{Y}(G)$ then $G_{1}(Y / G)=Z\left(\pi_{1}(Y / G)\right) \cong Z(G)$.

Proof. Let $g \in Z(G)$ then $g \in Z_{Y}(G)$. Since $Z(G)$ lies in a path component of $Z_{Y}(G)$ there is a path $\sigma: I \rightarrow Y$ such that $\sigma(0)=g, \sigma(1)=e$, and $\sigma(t) \in Z_{Y}(G)$ for all $t \in I$. Consider the homotopy $h_{t}(y)=\sigma(t) \cdot y$ for all $y \in Y$. For any $g^{\prime} \in G, h_{t}\left(\ell_{g^{\prime}}(y)\right)=\sigma(t) \cdot g^{\prime} \cdot y=g^{\prime} \cdot \sigma(t) \cdot y=$ $\ell_{g^{\prime}}\left(h_{t}(y)\right)$ since $\sigma(t) \in Z_{Y}(G)$; thus $h_{t}$ is fiber preserving by Theorem II.2. Now $h_{0}(y)=\sigma(0) \cdot y=g \cdot y=\ell_{g}(y)$ and $h_{1}(y)=\sigma(1) \cdot y=e \cdot y=y$. Thus $h_{t}$ is a homotopy from $\ell_{g}$ to $1_{Y}$ and by Theorem II.1, $\psi(g) \in$ $G_{1}(Y / G)$.

The following theorem is due to J. Siegel [12]. In this theorem $G$ need not be finite.

THEOREM II.5. Let $Y$ be a Lie group and $G$ any closed subgroup. If $p: Y \rightarrow Y / G$ is the quotient map, $p_{*} \pi_{i}(Y) \subset G_{i}(Y / G)$ for all $i$.

Proof. Consider the natural pairing $\mu: Y / G \times Y \rightarrow Y / G$. If $\alpha \epsilon$ $p_{*} \pi_{i}(Y)$ there is a map $f: S^{i} \rightarrow Y$ such that $\alpha=[p o f]$. Then the $\operatorname{map} \phi: Y / G \times S^{i} \stackrel{1 \times f}{\longrightarrow} Y / G \times Y \stackrel{\mu}{\longrightarrow} Y / G$ is an associated map for $\alpha$ and $\alpha \in G_{i}(Y / G)$ by Definition I.1.

CoROllaRY II.6. For $G$ a finite subgroup $G_{n}(Y / G)=\pi_{n}(Y / G)$ for $n>1$.

Proof. Consider the long exact sequence for the fibration $p: Y \rightarrow$ $Y / G$ :

$$
\cdots \longrightarrow \pi_{n}(Y) \stackrel{p_{*}}{\longrightarrow} \pi_{n}(Y / G) \stackrel{\partial}{\longrightarrow} \pi_{n-1}(G) \longrightarrow \cdots \text {. }
$$

For $n>1, \pi_{n-1}(G)=0$ since $G$ has the discrete topology. Then by exactness $p_{*}$ is onto and $G_{n}(Y / G)=\pi_{n}(Y / G)$.

It can now be shown that many factor spaces of Lie groups are 
$G$-spaces.

THEOREM II.7. If $Y$ is a compact simply connected Lie group and $G$ a finite subgroup contained in a torus $T$ in $Y$, then $Y / G$ is a G-space.

Proof. $Z(G) \subset T$ and thus in a path component of $Z_{Y}(G)$. By Theorem II.4, $G_{1}(Y / G)=Z(G)=\pi_{1}(Y / G)$ since $G$ must be abelian. That $G_{i}(Y / G)=\pi_{i}(Y / G)$ follows from Corollary II.6.

Definition II.8. $G$ is a $[p]$-subgroup of $Y$ for $p$ prime if $G$ is the direct sum of a finite number of copies of $Z_{p}$ (the integers, $\bmod p$ ).

Definition II.9. $Y$ is said to be without $p$-torsion is the cohomology groups of $Y$ do not contain any nonzero elements of order divisable by $p$.

CoROLlary II.10. If $Y$ is a compact connected Lie group without p-torsion and $G$ is a [p]-subgroup of $Y$ then $Y / G$ is a $G$-space.

Proof. By Theorem 3.2 of [2], $G$ lies on a torus in $Y$. Then $Y / G$ is a $G$-space by Theorem II.7.

COROLLARY II.11. If $Y$ is a compact simply connected Lie group and $G$ is of the form $Z_{p}$ or $Z_{p} \oplus Z_{p}$, then $Y / G$ is a $G$-space.

Proof. For $Y$ simply connected any group of the form $Z$ or $Z_{p} \oplus Z_{p}$ must lie on a torus.

\section{Calculations of evaluation subgroups.}

A. Orbit spaces of $S^{3}$. The following theorems calculate the evaluation subgroups for the orbit spaces of $S^{3}$ under the action of a binary polyhedral group. These spaces provide a nice demonstration of the use of Theorems II.3 and II.4.

DEFINITION III.1. $\langle l, m, n>$ will denote the binary polyhedral group generated by $R, S$, and $T$ and satisfying relations $R^{l}=S^{m}=$ $T^{n}=R S T$.

These groups will be finite in the cases $\langle 2,2, n\rangle,\langle 2,3,3\rangle$, $\langle 2,3,4\rangle$, and $\langle 2,3,5\rangle$ having order $4 n, 24,48$, and 120 respectively. The following classical result is due to H. S. M. Coxeter (see [2] or [3]). 
LEMMA III.2. The finite binary polyhedral groups are subgroups of $S^{3}$.

The orbit space $S^{3} /\langle 2,3,5\rangle$ is the dodecehedral space which provided the original counterexample to the first form of the Poincaré conjecture (see [1], p. 217).

THEOREM III.3. $G_{1}\left(S^{3} / G\right)=Z_{2}$ for $G=\langle 2,2, n\rangle, n \geqq 2,\langle 2,3,3\rangle$, $<2,3,4>$ and $<2,3,5\rangle$.

Proof. In these cases $Z(G)=Z_{2}$ which can be taken as the subgroup $\{1,-1\}$ of the quaternians. But $\{1,-1\}$ is also the center of $S^{3}$ so by Theorem II.3, $Z_{2} \subset G_{1}\left(S^{3} / G\right)$. By Theorem I.8, $G_{1}\left(S^{3} / G\right) \subset$ $Z(G)$, thus $G_{1}\left(S^{3} / G\right)=Z(G)=Z_{2}$.

THEOREM III.4. $\left.G_{1}\left(S^{3} /<2,2,1\right\rangle\right)=\langle 2,2,1\rangle=Z_{4}$.

Proof. The group $\langle 2,2,1\rangle$ can be taken to be the subgroup $\{1, i,-i,-1\}$ of the quaternians. The centrilizer of $\langle 2,2,1\rangle$ in $S^{3}$ is the set of quaternians of the form $a+b i$, a copy of $S^{1}$. In particular $\left.Z_{S^{3}}(<2,2,1\rangle\right)$ is path connected. Then, by Theorem II.4, $G_{1}\left(S^{3} /<2,2,1>\right)=Z_{4}$.

THEOREM III.5. For $G$ any of the binary polyhedral groups $G_{n}\left(S^{3} / G\right)=\pi_{n}\left(S^{3} / G\right) \cong \pi_{n}\left(S^{3}\right)$ for $n>1$.

Proof. This is immediate from Corollary II.6 and the fact that, since $G$ is finite, $S^{3}$ is the universal covering space of $S^{3} / G$.

B. Complex projective spaces. Let $C P^{n}, n \geqq 1$ denote complex projective $n$-space. Let $p: S^{2 n+1} \rightarrow C P^{n}$ denote the usual fibration with fiber $S^{1}$. The base point of $S^{2 n+1}$ will be taken as $(1,0,0, \cdots, 0)$ and $S^{1}$ will be embedded in $S^{2 n+1}$ by $i(z)=(z, 0,0, \cdots, 0)$.

TheOREM III.6. $G_{2}\left(C P^{n}\right)=0$ for all $n$.

Proof. Assume $\phi: S^{2} \times C P^{n} \rightarrow C P^{n}$ is an associated map for $\alpha \epsilon$ $\pi_{2}\left(C P^{n}\right)$. Consider the following diagram:

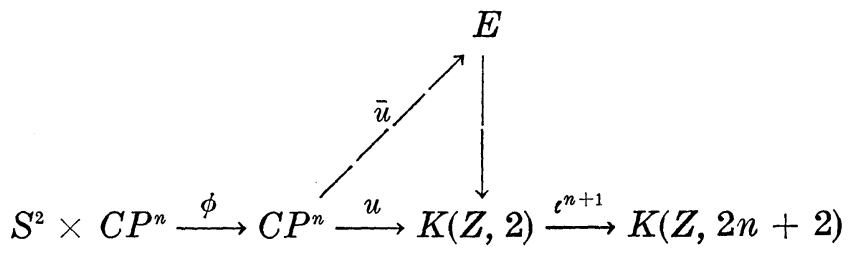


where $\iota \in H^{2}(Z, 2 ; Z)$ is the fundamental class, $E$ is induced from the path fibration by $\iota^{n+1}, u \in H^{2}\left(C P^{n}\right)$ is a generator and $\bar{u}$ a lift of $u$. Let $\sigma \in H\left(S^{2}\right)$ be a generator and $\alpha^{*} u=m \sigma$. Since $\bar{u}$ lifts $u,(u \phi)^{*} \iota^{n+1}=$ 0 . By direct calculation we have

$$
(u \phi)^{*} \iota^{n+1}=\left[(u \phi)^{*} \iota\right]^{n+1}=(m \sigma \otimes 1+1 \otimes u)^{n+1}=(n+1) m \sigma \otimes u^{n} .
$$

The second equality follows from the fact that $\phi \mid S^{2} \times^{*}=\alpha$ and $\left.\phi\right|^{*} \times C P^{n}=1$, the others are standard. But $u^{n} \in H^{2 n}\left(C P^{n}\right)$ is a generator, so $(n+1) m \sigma \otimes u^{n}=0$ implies $m=0$. Thus $\alpha$ is null homotopic. This proof was suggested by the referee.

In [1] it is shown that for all $n, P_{r}\left(C P^{n}\right) \subset p_{*} P_{r}\left(S^{2 n+1}\right)$ if $r>2$. In particulas $C P^{3}$ is a $W$-space. Since $G_{2}\left(C P^{3}\right) \neq \pi_{2}\left(C P^{3}\right), C P^{3}$ is an example of a finite dimensional space which is a $W$-space but not a $G$-space. Since $G_{r}\left(C P^{n}\right) \subset P_{r}\left(C P^{n}\right)$ the above result implies:

CoRollaRY III.7. $\quad G_{2 n+1}\left(C P^{n}\right) \subset P_{2 n+1}\left(C P^{n}\right) \subset p_{*} P_{2 n+1}\left(S^{2 n+1}\right)=2 Z$ for $n \neq 2,3$.

Using Theorem II.5 a lower bound for $G_{2 n+1}\left(C P^{n}\right)$ can be obtained. A new representation of $C P^{n}$ will be needed. Let $U(n)$ be the space of all $n \times n$ unitary matrices. Let $i: U(n) \times S^{1} \rightarrow U(n+1)$ be given by

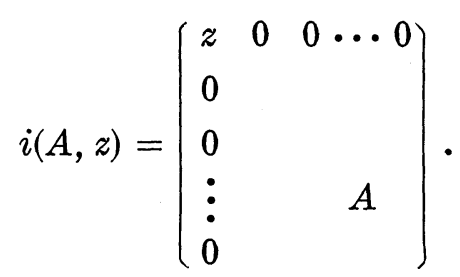

Using 7.3 of [17] it is easy to check that $C P^{n}=U(n+1) / U(n) \times S^{1}$ and there is a fibration $U(n) \stackrel{i}{\longrightarrow} U(n+1) \stackrel{p}{\longrightarrow} C P^{n}$.

Theorem III.8. $n ! Z \subset G_{2 n+1}\left(C P^{n}\right)$.

Proof. The above fibration yields an exact sequence

$$
\cdots \longrightarrow \pi_{2 n+1}(U(n+1)) \stackrel{p_{*}}{\longrightarrow} \pi_{2 n+1}\left(C P^{n}\right) \stackrel{\partial}{\longrightarrow} \pi_{2 n}\left(U(n) \times S^{1}\right) \longrightarrow \pi_{2 n}(U(n+1)) \text {. }
$$

Now $\pi_{2 n+1}\left(C P^{n}\right)=Z$ and $\pi_{2 n}\left(U(n) \times S^{1}\right)=\pi_{2 n}(U(n))=Z_{n !}$. By the Bott Periodicity Theorem $\pi_{2 n}(U(n+1))=0$ and $\left.\pi_{2 n+1}(U+1)\right)=Z$. The above segment of the exact sequence then reduces to $Z \stackrel{p_{*}}{\longrightarrow} Z \stackrel{\hat{\partial}}{\longrightarrow}$ $Z_{n !} \longrightarrow 0$. Thus $\operatorname{Ker} \partial=n ! Z$ and by exactness $\operatorname{Im} p_{*}=n ! Z$. Then by Theorem II.5, $n ! Z \subset G_{2 n+1}\left(C P^{n}\right)$. 
C. The Stiefel manifolds. In this section some evaluation subgroups of the Stiefel manifolds are computed and the inplications of the evaluation subgroups on the James number is explored. A number of these groups can be shown to be nonzero by checking the boundary homomorphisms of the various fibrations used by Paechter (see [11]) in his extensive calculations of the homotopy groups of the real Stiefel manifolds. This technique however yields a complete calculation for very few groups. The Lie groups of the orthogonal, unitary, and symplectic $n \times n$ matrices will be denoted respectively by $O(n), U(n)$, and $\operatorname{Sp}(n)$. There are natural embeddings $O(n) \rightarrow O(m), U(n) \rightarrow U(m)$, $\operatorname{Sp}(n) \rightarrow \operatorname{Sp}(m)$ for $n \leqq m$.

Definition III.13. The real, complex, and quaternic Stiefel manifolds are defined respectively by

$$
V_{n, k}=O(n) / O(n-k), W_{n, k}=U(n) / U(n-k),
$$

and

$$
X_{n, k}=\operatorname{Sp}(n) / \operatorname{Sp}(n-k) \text {. }
$$

The notation $O_{n, k}$ will be used for any of these manifolds; in this case $d$ will denote the dimension of the scalar field over the reals. There is a fibration $O_{m-1, k-1} \longrightarrow O_{m, k} \longrightarrow S^{d m-1}$. The following definition, due to I. M. James (see [9] and [13]), uses the boundary homomorphism of the long exact homotopy sequence of this fibration. $\iota_{d m-1} \in$ $\pi_{d m-1}\left(S^{d m-1}\right)$ will denote the class of the identity.

DEFINITION III.14. The James number $O\{m, k\}$ is defined to be the order of $\partial\left(l_{d m-1}\right)$ in $\pi_{d m-2}\left(O_{m-1, k-1}\right)$ for $2 \leqq k \leqq m$ and in the real case $m \geqq 3$. By convention $O\{m, k\}=0$ if $\partial\left(\iota_{d m-1}\right)$ is of infinite order and $O\{m, k\}=1$ if $k=1$ or in the real case if $m=2$.

DEFINITION III.15. If $2 \leqq k \leqq m$ where $m \geqq 3$ in the real case, let $0<m, k>$ be the order of $G_{d m-2}\left(O_{m-1, k-1}\right)$. By convention $0<m, k>=$ 0 if $G_{d m-2}\left(O_{m-1, k-1}\right)$ is infinite and $0<m, k>=1$ if $k=1$ or in the real case if $m=2$.

ThEOREM III.16. (a) If $0<m, k>\neq 0$, then $O\{m, k\}$ divides $0<m, k>$. 0 or 1 .

(b) If $0<m, k>=0$ and $G_{d m-2}\left(O_{m-1, k-1}\right)$ is torsion free, $O\{m, k\}=$

(c) If $O\{m, k\}=0$, then $0<m, k>=0$.

Proof. By Theorem I.2, $\partial\left(\iota_{d m-1}\right)$ is in $G_{d m-2}\left(O_{m-1, k-1}\right)$ and thus if $0<m, k>\neq 0$ the order of the group generated by $\partial\left(c_{d m-1}\right)$ must 
divide the order of $G_{d m-2}\left(O_{m-1, k-1}\right)$ proving (a). If $0<m, k>=0$ and $G_{d m-2}\left(O_{m-1, k-1}\right)$ is torsion free, $\partial\left(\iota_{d m-2}\right)$ must be 0 or generate an infinite group, thus $O\{m, k\}=0$ or 1 proving (b). Part (c) is clear from Theorem I.2.

Theorem III.17. If $k \geqq 1, m$ odd, then $G_{m}\left(V_{m+1, k}\right)$ is infinite.

Proof. $V\{m, k\}=0$ for $k \geqq 2, m$ odd, by $\S 25.6$ in [17]. Then by Theorem III.16, (c) $V\langle m, k\rangle=0$ and $G_{m-2}\left(V_{m-1, k-1}\right)$ is infinite and the result follows by a simple shift of indices.

THEOREM III.18. $G_{2 i+1}\left(W_{n, k}\right)$ is infinite for $n-k=2,2 \leqq i \leqq$ $n-1$.

Proof. Consider the fibration $U(n-k) \stackrel{i}{\longrightarrow} U(n) \stackrel{p}{\longrightarrow} W_{n, k}$. The homotopy exact sequence contains

$$
\begin{aligned}
& \cdots \longrightarrow \pi_{2 i+1}(U(n-k)) \stackrel{i_{*}}{\longrightarrow} \pi_{2 i+1}(U(n)) \stackrel{p_{*}}{\longrightarrow} \pi_{2 i+1}\left(W_{n, k}\right) \\
& \stackrel{\partial}{\longrightarrow} \pi_{2 i}(U(n-k)) \longrightarrow \cdots
\end{aligned}
$$

By $\S 24.5$ in [17], $U(2)$ is homeomorphic to $S^{3} \times S^{1}$. Thus for $i \geqq 2$, $\pi_{2 i+1}(U(n-k))=\pi_{2 i+1}\left(S^{3}\right)$ is finite (see p. 318 in [9]). For $i \leqq n-1$, $\pi_{2 i+1}(U(n))=Z$ since it is in the stable range of the Bott Periodicity Theorem. But then $i_{*}$ must be trivial and $p_{*}$ a monomorphism. But then $p_{*} \pi_{2 n-1}(U(n))$ is infinite and so is $G_{2 i+1}\left(W_{n, k}\right)$ by Theorem II.5.

The first nonvanishing homotopy group of $V_{n, k}$ occurs in dimension $n-k$ and is given by $\pi_{n-k}\left(V_{n, k}\right)=Z$ if $n-k$ is even or $k=1$ and $\pi_{n-k}\left(V_{n, k}\right)=Z_{2}$ otherwise (see $\S 25.6$ in [17]).

THEOREM III.19.

$$
G_{n-k}\left(V_{n, k}\right)= \begin{cases}Z & k=1, n=2,4, \text { or } 8 \\ 2 Z & k=1, n \text { even, } n \neq 2,4, \text { or } 8 \\ 0 & n-k \text { even } \\ Z_{2} & k>1, n-k=1 \text { or } 3 .\end{cases}
$$

Proof. For $k=1, V_{n, 1}=S^{n-1}$ and the first two results follow from Theorem I.9. If $n-k$ is even the Hurewicz homomorphism is an isomorphism in dimension $n-k$ and by Theorem 5.1 in [7], $G_{n-k}\left(V_{n, k}\right)$ must be torsion. But $\pi_{n-k}\left(V_{n, k}\right)$ is torsion free for $n-k$ even and thus $G_{n-k}\left(V_{n, k}\right)=0$. When $n-k=1, k>1, V_{n, k}=S O(n)$, the special orthogonal group and $V_{n, k}$ is a $G$-space. Thus $\pi_{n-k}\left(V_{n, k}\right)=Z_{2}$ for $n-k=1$. Now assume $n-k=3$ and consider the fibration 


$$
O(n-k) \longrightarrow O(n) \longrightarrow V_{n, k}
$$

There is a long exact sequence containing

$$
\cdots \longrightarrow \pi_{3}(O(n)) \stackrel{p_{*}}{\longrightarrow} \pi_{3}\left(V_{n, k}\right) \stackrel{\partial}{\longrightarrow} \pi_{2}(O(n-k)) \longrightarrow \cdots .
$$

Since $O(n-k)$ is a group, $\pi_{2}(O(n-k))=0$ and $p_{*}$ is onto. But then by Theorem II.5, $G_{n-k}\left(V_{n, k}\right)=\pi_{n-k}\left(V_{n, k}\right)=Z_{2}$ for $n-k=3, k>1$.

\section{REFERENCES}

1. M. G. Barratt, I. M. James and N. Stein, Whitehead products and projective spaces,

J. Math. Mech., 9 (1960), 813-819.

2. Armand Borel, Commutative subgroups and torsion in compact Lie groups, Bull. Amer. Math. Soc., 66 (1960), 285-288.

3. H. S. M. Coxeter, Quaternians and reflections, Amer. Math. Monthly, 53 (1946), 136146.

4. H. S. M. Coxeter and W. O. J. Moser, Generators and relations for discrete groups, 2nd ed., Ergebnisse der Mathematik und ihrer Grenzgebiete, Neue Folge, Band 14, Springer-Verlag, Berlin, 1966.

5. T. Ganea, Cyclic homotopics, Illinois J. Math., 12 (1968), 1-4.

6. Daniel H. Gottlieb, A certain subgroup of the fundamental group, Amer. J. Math., 87 (1965), 840-856.

7. — Evaluation subgroups of homotopy groups, Amer. J. Math., 91 (1969), $729-755$.

8. - On fibre spaces and the evaluation map, Ann. of Math., (2) 87 (1968), 4255 .

9. Sze-Tsen Hu, Homotopy theory, Academic Press, New York, 1959.

10. I. M. James, Cross-sections of Stiefel manifolds, Proc. London Math. Soc., 8 (1958), $36-47$.

11. G. F. Paechter, The groups $\pi_{r}\left(V_{n, m}\right)(I)$, Quart. J. Math. Oxford Ser., (2) 7 (1956), 249-268.

12. H. Seifert and W. Trefall, Lehrbuch der Topologie, B. G. Teubner, Leipzig, 1934.

13. Jerrold Siegel, G-spaces, W-spaces, and H-spaces, Pacific J. Math., 31 (1970), 209214.

14. Francois Sigrist, Sur les nombres de James des variétiés de Stiefel complexes, Illinois J. Math., 13 (1969), 198-201.

15. Edwin H. Spanier, Algebraic topology, McGraw-Hill Book Company, New York, 1966.

16. J. Stasheff, On homotopy abelian H-spaces, Proc. Cambridge Philos. Soc., 57 (1961), 734-745.

17. Norman Steenrod, The topology of fibre bundles, Princeton University Press, Princeton, 1951.

Received May 5, 1971. This paper constituted part of the author's doctoral thesis written at Purdue University under Daniel H. Gottlieb.

FAIRFIELd UNIVERSITY 



\section{PACIFIC JOURNAL OF MATHEMATICS}

\section{EDITORS}

\section{H. SAMELSON}

Stanford University

Stanford, California 94305

\section{R. HOBBY}

University of Washington

Seattle, Washington 98105

\section{J. DugundjI}

Department of Mathematics University of Southern California Los Angeles, California 90007

RICHARD ARENS

University of California

Los Angeles, California 90024

\section{ASSOCIATE EDITORS}
E. F. BECKENBACH
B. H. NeumanN
F. WOLF
K. YOSHIDA

\section{SUPPORTING INSTITUTIONS}

\author{
UNIVERSITY OF BRITISH COLUMBIA \\ CALIFORNIA INSTITUTE OF TECHNOLOGY \\ UNIVERSITY OF CALIFORNIA \\ MONTANA STATE UNIVERSITY \\ UNIVERSITY OF NEVADA \\ NEW MEXICO STATE UNIVERSITY \\ OREGON STATE UNIVERSITY \\ UNIVERSITY OF OREGON \\ OSAKA UNIVERSITY
}

\author{
UNIVERSITY OF SOUTHERN CALIFORNIA \\ STANFORD UNIVERSITY \\ UNIVERSITY OF TOKYO \\ UNIVERSITY OF UTAH \\ WASHINGTON STATE UNIVERSITY \\ UNIVERSITY OF WASHINGTON \\ AMERICAN MATHEMATICAL SOCIETY \\ NAVAL WEAPONS CENTER
}

The Supporting Institutions listed above contribute to the cost of publication of this Journal, but they are not owners or publishers and have no responsibility for its content or policies.

Mathematical papers intended for publication in the Pacific Journal of Mathematics should be in typed form or offset-reproduced, (not dittoed), double spaced with large margins. Underline Greek letters in red, German in green, and script in blue. The first paragraph or two must be capable of being used separately as a synopsis of the entire paper. The editorial "we" must not be used in the synopsis, and items of the bibliography should not be cited there unless absolutely necessary, in which case they must be identified by author and Journal, rather than by item number. Manuscripts, in duplicate if possible, may be sent to any one of the four editors. Please classify according to the scheme of Math. Rev. Index to Vol. 39. All other communications to the editors should be addressed to the managing editor, Richard Arens, University of California, Los Angeles, California, 90024.

50 reprints are provided free for each article; additional copies may be obtained at cost in multiples of 50 .

The Pacific Journal of Mathematics is published monthly. Effective with Volume 16 the price per volume (3 numbers) is $\$ 8.00$; single issues, $\$ 3.00$. Special price for current issues to individual faculty members of supporting institutions and to individual members of the American Mathematical Society: $\$ 4.00$ per volume; single issues $\$ 1.50$. Back numbers are available.

Subscriptions, orders for back numbers, and changes of address should be sent to Pacific Journal of Mathematics, 103 Highland Boulevard, Berkeley, California, 94708.

PUBLISHED BY PACIFIC JOURNAL OF MATHEMATICS, A NON-PROFIT CORPORATION

Printed at Kokusai Bunken Insatsusha (International Academic Printing Co., Ltd.), 270, 3-chome Totsuka-cho, Shinjuku-ku, Tokyo 160, Japan. 


\section{Pacific Journal of Mathematics}

\section{Vol. 42, No. $3 \quad$ March, 1972}

Catherine Bandle, Extensions of an inequality by Pólya and Schiffer for vibrating membranes ................................ 543

S. J. Bernau, Topologies on structure spaces of lattice groups.......... 557

Woodrow Wilson Bledsoe and Charles Edward Wilks, On Borel product measures .......................................

Eggert Briem and Murali Rao, Normpreserving extensions in subspaces of

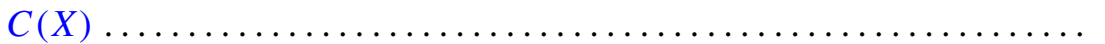

Alan Seymour Cover, Generalized continuation.................. 589

Larry Jean Cummings, Transformations of symmetric tensors .......... 603

Peter Michael Curran, Cohomology of finitely presented groups .......... 615

James B. Derr and N. P. Mukherjee, Generalized quasicenter and

hyperquasicenter of a finite group ...................... 621

Erik Maurice Ellentuck, Universal cosimple isols .................. 629

Benny Dan Evans, Boundary respecting maps of 3-mainfolds .......... 639

David F. Fraser, A probabilistic method for the rate of convergence to the

Dirichlet problem .................................. 657

Raymond Taylor Hoobler, Cohomology in the finite topology and Brauer

groups ..................................... 667

Louis Roberts Hunt, Locally holomorphic sets and the Levi form ........ 681

B. T. Y. Kwee, On absolute de la Vallée Poussin summability............ 689

Gérard Lallement, On nilpotency and residual finiteness in semigroups .... 693

George Edward Lang, Evaluation subgroups of factor spaces........... 701

Andy R. Magid, A separably closed ring with nonzero torsion pic ....... 711

Billy E. Rhoades, Commutants of some Hausdorff matrices ............. 715

Maxwell Alexander Rosenlicht, Canonical forms for local derivations . . . . 721

Cedric Felix Schubert, On a conjecture of L. B. Page ................ 733

Reinhard Schultz, Composition constructions on diffeomorphisms of $S^{p} \times S^{q}$

J. P. Singhal and H. M. (Hari Mohan) Srivastava, A class of bilateral generating functions for certain classical polynomials ....

Richard Alan Slocum, Using brick partitionings to establish conditions which insure that a Peano continuum is a 2-cell, a 2-sphere or an annulus...

James F. Smith, The p-classes of an $H^{*}$-algebra ...

Jack Williamson, Meromorphic functions with negative zeros and positive

poles and a theorem of Teichmuller ................. 